\title{
Practical first-line management of renal cell carcinoma in a community practice
}

\author{
Henry Jacob Conter, MD, MSc
}

William Osler Health System, Brampton, ON, Canada

Cite as: Can Urol Assoc J 2016;10(11-12Supp17):S239-41.

http://dx.doi.org/10.5489/cuaj.4295

\section{Abstract}

Sunitinib is an oral receptor tyrosine kinase inhibitor (TKI) that targets signalling by vascular endothelial growth factor receptors (VEGFRs). The standard sunitinib dosing schedule for metastatic renal cell carcinoma (mRCC) is $50 \mathrm{mg}$ for four weeks (28 days) of treatment, followed by a two-week (14-day) break from treatment (four/two schedule). However, this schedule is associated with toxicities that can limit the patient's health-related quality of life (HRQOL) and impede treatment compliance. Given the generally incurable nature of $\mathrm{mRCC}$ and the toxicity associated with therapy, treatment strategies should focus on achieving long-term response, preserving $\mathrm{HRQOL}$, and minimizing treatment-related toxicity. The William Osler Cancer Clinic in Brampton, $\mathrm{ON}$, has instituted an alternative schedule of sunitinib treatment as our standard dosing strategy, involving two weeks of treatment, followed by a oneweek break (two/one schedule), to minimize toxicity and improve HRQOL among their patients with mRCC.

\section{Introduction}

Sunitinib is an oral receptor tyrosine kinase inhibitor (TKI) that targets signalling by vascular endothelial growth factor receptors (VEGFRs). In the pivotal randomized, phase 3 trial, sunitinib was associated with significantly longer progression-free survival (PFS) than interferon-alfa (IFN-alfa) in patients with previously untreated metastatic renal cell carcinoma (mRCC) (11 months vs. five months; $\mathrm{p}<0.001){ }^{1}$ Along with the anti-VEGFR TKI pazopanib and the mammalian target of rapamycin (mTOR) inhibitor temsirolimus, sunitinib has since become a standard of care in the first-line treatment of mRCC. Although these targeted agents have demonstrated PFS benefit, durable responses are rare, and most patients with mRCC eventually experience disease progression. $^{2}$
With respect to medical outcomes, survival is generally at the top of the hierarchy, followed by time to recovery and return to normal activity, and then sustainability of health or recovery and nature of recurrences (Fig. 1). ${ }^{3}$ However, with conditions such as mRCC, which are associated with a relatively poor prognosis and therapies with toxicities that may limit health-related quality of life (HRQOL), treatments may have limited effect on survival, but may be differentiated based on their ability to improve timely provision of care, reduce discomfort, and minimize recurrence. ${ }^{3}$ While overall survival (OS) remains an important outcome, and PFS holds some meaning to the patient, the value of HRQOL to the patient cannot be underestimated. Outcomes such as avoidance of clinic visits, trips to the emergency room, and multiple rounds of tests take on greater importance for patients with incurable diseases such as mRCC. In the PISCES study comparing pazopanib and sunitinib in 168 patients with mRCC, significantly more patients preferred pazopanib $800 \mathrm{mg} /$ day for 10 weeks over sunitinib $50 \mathrm{mg}$ per day in a four/two schedule $(70 \%$ vs. $22 \%$; $<<0.001)$; less fatigue and better overall HRQOL were identified as the main reasons for this preference. ${ }^{4}$ This study was key in showing that differences in symptomatic toxicities between two drugs are meaningful to patients.

The standard sunitinib dosing schedule is $50 \mathrm{mg}$ for four weeks (28 days) of treatment, followed by a two-week (14day) break from treatment (four/two schedule). ${ }^{5}$ This dose and schedule were selected based on preclinical trial data showing that toxicities were manageable at this level. ${ }^{6}$ However, in clinical practice, adverse effects (AEs) such as fatigue, diarrhea, nausea, mucositis/stomatitis, hypertension, and hand-foot syndrome are common, often increasing throughout each cycle and peaking in the final two weeks of treatment. AEs often lead to dose reductions or interruptions, ${ }^{1}$ which can have important consequences on outcomes, as data suggest that maximizing the dose intensity of sunitinib is key to its efficacy. ${ }^{7}$ Alternative dosing schedules 


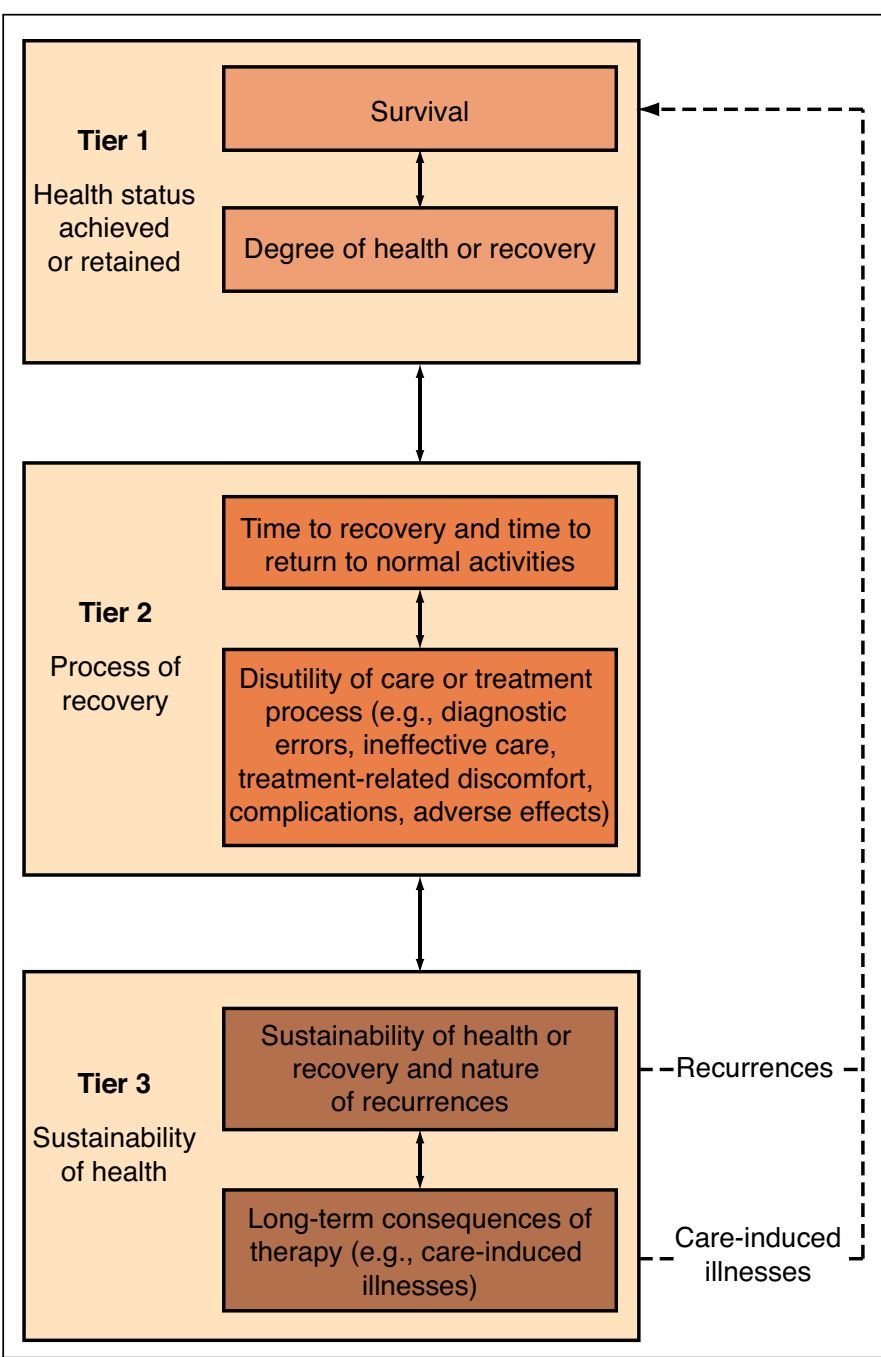

Fig. 1. Outcomes measures hierarchy for healthcare. ${ }^{3}$

for sunitinib have, therefore, been explored, with the aim of improving drug tolerance while maintaining dose intensity. One of the most common alternative schedules is administration of sunitinib for two weeks ( 14 days), followed by one week (seven days) off (two/one schedule).

In a retrospective analysis of patients with mRCC in China, data were reviewed from 108 patients treated with first-line sunitinib in one of three regimens - a four/two schedule $(n=50)$, a transitional schedule starting with a four/ two schedule and switching to a two/one schedule due to toxicity $(n=26)$, and an initial two/one schedule $(n=32) .{ }^{8}$ AEs, including hand-foot syndrome, fatigue, diarrhea, and bone marrow suppression, were significantly less common in both the transitional four/two to two/one schedule and the initial two/one schedule than in the four/two schedule $(p<0.05)$. HRQOL, measured by Functional Assessment of Cancer Therapy-Kidney Symptom Index-19 (FKSI-19) scores, did not differ significantly among the three groups during the first two treatment cycles; however, HRQOL declined significantly less during Cycles 3-9 in the group treated initially with a two/one schedule, than among those treated with either a four/two schedule or the transitional schedule. Median PFS was longer in the group treated with an initial two/one schedule (11.2 months) than in either the four/ two schedule (9.4 months) or the transitional schedule (9.5 months) groups ( $p=0.030)$. While limited by its retrospective design and small sample size, this study showed that a modified dosing schedule with shorter on/off treatment periods may be a useful approach to minimizing drug toxicity and maximizing $\mathrm{HRQOL}$ while maintaining efficacy.

Recently, a multicentre, randomized, open-label, phase 2 trial randomly assigned patients with mRCC to first-line treatment with either a four/two schedule $(n=36)$ or a two/ one schedule $(n=38)$ of sunitinib. ${ }^{9}$ The primary endpoint of failure-free survival (FFS) at six months was higher in the two/one schedule than in the four/two schedule $(63 \%$ vs. $44 \%)$, as was FFS (7.6 months vs. 6.0 months; $p=0.029$ ). The two/one schedule was associated with less frequent treatment-emergent $\mathrm{AEs}$, including stomatitis, rash, and hand-foot syndrome. Objective response rate and time to progression were not compromised by the two/one schedule, and were numerically higher than in the four/two group. While the results of this study are limited by a small sample size and the open-label design, they provide reassurance that patients may be initiated on a two/one schedule to improve tolerability without compromising efficacy.

Resource utilization is another factor that can influence treatment of RCC in the community setting. In the COMPARZ study comparing pazopanib with sunitinib in the first-line setting, medical resource utilization was evaluated as a secondary endpoint. ${ }^{10}$ This included medical office visits not related to the study, telephone consultations, number of days in hospital, and emergency room (ER) visits. Significant differences favoured pazopanib for two comparisons on medical resource utilization (telephone consultations and ER visits), largely due to the lower incidence of toxicities compared with sunitinib. Given the reduction in toxicities demonstrated with a two/one schedule for sunitinib, it is reasonable to suggest that the alternative dosing strategy would translate to a reduction in medical resource utilization. It is important to note that the standard patient followup of every six weeks is still generally maintained with the alternative dosing schedule. With a four/two schedule, this followup generally takes place at the end of the two weeks off sunitinib treatment. With a two/one schedule, patients are still followed every six weeks - at the end of the second one-week break from dosing. Many clinicians will see the patient after the first three-week cycle to ensure that patients are tolerating treatment, but after that, the six-week monitoring schedule can be maintained.

While some academic centres have described a rigid algorithmic approach to dose-adjustment of sunitinib involving 
toxicity management and dose-escalation, in the resourceconstrained community practice setting, minimizing variation between patients can lead to improved care. ${ }^{11}$ In such a setting, nurses are often responsible for many different diseases with numerous treatments across each disease spectrum. Therefore, maneuvering through multiple algorithms for various treatments can be a hindrance to efficient practice and increase the incidence of errors. In the William Osler Cancer Clinic, standardization of treatment regimens and the development of care pathways have decreased overall resource utilization, questions among nurses, and errors, while increasing comfort among allied healthcare providers, especially nurses and pharmacists. With the ability to anticipate the physician's treatment strategy and expectations, allied healthcare providers have a greater understanding of each step of the care pathway, including how to make arrangements for imaging and bloodwork, timing of followup, and toxicity management strategies. In contrast, an algorithmic system often allows for a "dealer's choice" strategy, with less standardization of the treatment pathway.

\section{Conclusion}

Although efficacy remains the primary goal of treatment for patients with mRCC, HRQOL outcomes are meaningful to patients, and their importance should not be underestimated when selecting a treatment. For the first-line treatment of patients with $\mathrm{mRCC}$, the standard schedule of sunitinib follows a four/two schedule, which has been associated with treatment-emergent AEs that can impact patients' $\mathrm{HRQOL}$ and, in some cases, limit their ability to remain on treat- ment at an optimal dose. At the William Osler Cancer Clinic in Brampton, an alternative two/one schedule for first-line sunitinib treatment was chosen based on what patients believe are most important with respect to their care, including improved tolerability with no loss in efficacy.

\section{References}

1. Motzer RJ, Hutson TE, Tomczak $P$, et al. Sunitinib vs. interferon-alfa in metastatic renal cell carcinoma. N Engl J Med 2007;356:115-24. https://doi.org/10.1056/NEJMoa065044

2. Albiges $L$, Oudard $S$, Negrier $S$, et al. Complete remission with tyrosine kinase inhibitors in renal cell carcinoma. J Clin Oncol 2012;30:482-7. https://doi.org/10.1200/JC0.2011.37.2516

3. Porter ME. What is value in healthcare? N Engl J Med 2010;363:2477-81. htrps://doi.org/10.1056/ NEJMp 1011024

4. Escudier B, Porta C, Bono P, et al. Randomized, controlled, double-blind, cross-over trial assessing treatment preference for pazopanib vs. sunitinib in patients with metastatic renal cell carcinoma: PISCES Study. J Clin Oncol 2014;32:1412-8. https://doi.org/10.1200/JC0.2013.50.8267

5. Sutent ${ }^{\circledR}$ (Sunitinib capsules). Product Monograph. Kirkland, QC: Pfizer Canada Inc., December 3, 2014.

6. Faivre S, Delbaldo C, Vera K, et al. Safety, pharmacokinetic, and antitumour activity of SU1 1248, a novel oral multitarget tyrosine kinase inhibitor, in patients with cancer. J Clin Oncol 2006;24:25-35. https://doi.org/10.1200/JC0.2005.02.2194

7. Houk BE, Bello CL, Poland B, et al. Relationship between exposure to sunitinib and efficacy and tolerability endpoints in patients with cancer: Results of a pharmacokinetic/pharmacodynamic meta-analysis. Cancer Chemother Pharmacol 2010;66:357-71. hitps://doi.org/10.1007/s00280-009-1170-y

8. Pan $X$, Huang $H$, Huang $Y$, et al. Sunitinib dosing schedule two/one improves tolerability, efficacy, and health-related quality of life in Chinese patients with metastatic renal cell carcinoma. Urol Oncol 2015;33:268.e9-15. https://doi.org/10.1016/j.urolonc.2015.03.008

9. Lee JL, Kim MK, Park I, et al. Randomized, phase 2 trial of sunitinib four weeks on and two weeks off vs. two weeks on and one week off in metastatic, clear-cell type renal cell carcinoma: RESTORE trial. Ann Oncol 2015;26:2300-5. https://doi.org/10.1093/annonc/mdv357

10. Motzer RJ, Hutson TE, Cella D, et al. Pazopanib vs. sunitinib in metastatic renal cell carcinoma. N Engl J Med 2013;369:722-31. https://doi.org/10.1056/NEJMoal303989

11. Magahanães AL, Erdmann AL, Silva EL, et al. Lean thinking in health and nursing: An integrative literature review. Rev Lat Am Enfermagem 2016;24:e2734. https://doi.org/10.1590/1518-8345.0979.2734 